\title{
尿毒症患者体液内中分子组分的多级色谱 分离比较及质谱分析"
}

储结根 刘晓航 袁 直 ${ }^{* *}$ 何炳林

(吸附分离功能高分子材料国家重点实验室, 南开大学高分子化学研究所, 天津 300071)

\begin{abstract}
摘要采用凝胶色谱、离子交换色谱以及反相高效液相色谱法, 将尿毒症患者的尿样 和血样以及正常人的尿样和血样进行了多级分离和比较. 通过凝胶色谱法, 从正常人 的尿液以及尿毒症血清和尿液中分离出相对于正常人血清具有异常高浓度的 A, B 2 个 中分子组分. 不同试样来源的 A 峰中分子物的离子交换色谱分离结果证明, A-3 亚峰中 分子物, 是尿毒症患者因肾衰难以通过尿液将其排除故而滞留在血清内的重要成分, 正常人却可以通过尿液将其排于体外. 将来自尿毒症血清及正常人尿液的 A-3 亚峰中 分子物经脱盐处理后, 进行了基质辅助激光解吸电离-飞行时间质谱分析, 证明 A-3 组 分由分子量分别为 $839,873,1007,1106,1680,2015$ 的 6 种化合物组成. 最后, 采用反相 高压液相色谱法对 A-3 亚峰中分子组分进行了进一步分离, 得到了 6 个只含单一中分子 化合物成分的组分. 至此, A-3 组分内的中分子物得到了彻底的分离和纯化, 为进一步 确定其结构以及病理生理作用奠定了基础.
\end{abstract}

\section{关键词 尿毒症 中分子物 反相高压液相色谱 基质辅助激光解吸电离-飞行时间质谱}

尿毒症中分子物是滞留在患者体内且血液透析疗法难以将其清除的重要毒素 ${ }^{[1]}$. 滞留的 中分子物和患者的许多症状密切相关 ${ }^{[2]}$. 然而对尿毒症中分子物的病理生理机制的认识尚极 为有限. 制约尿毒症中分子物毒性作用机理的研究和认识的一个重要原因就是尿毒症中分子 物是一群成分极为复杂的化合物, 其分离和纯化工作极其困难. 前人的工作中, 仅有几例报道 了对尿毒症中分子组分内很少几种化合物, 进行了分离纯化和结构鉴定 ${ }^{[3,4]}$. 许多分离工作都 只得到了中分子物的混合组分 ${ }^{[5 \sim 7]}$. 以这些混合物组分去研究中分子物的毒性作用, 难以得到 深入和清楚的认识. 首先, 以这些混合物组分进行的研究, 难以说明尿毒症的某一症状究竟是 由那种或那几种中分子化合物造成的, 更难说明其致病机理. 另一方面, 混合组分也制约了对 中分子物的化学组成和结构的鉴定工作, 不清楚中分子物的组成和结构, 就不能从分子水平 上真正地阐释其致病机理.

以前我们曾报道了采用凝胶色谱法对正常人尿液、尿毒症血清以及正常人血清的分离, 从 正常人尿液, 尿毒症血清中得到了 A, B 2 个中分子组分, 并采用离子交换色谱法对不同来源 的 $\mathrm{B}$ 峰中分子物进行了分离和比较, 得到了一些重要的结论 ${ }^{[8]}$. 本文在以前工作的基础上, 对

2000-12-12 收稿, 2001-11-26 收修改稿

* 国家自然科学基金(批准号: 59873011)、国家重点基础研究发展规划项目 (G1999064707)、跨世纪优秀人才培养计划基 金和高校博士点专项科研基金(批准号: 98005503)资助项目

**联系人, E-mail: zhiy@ public.tpt.tj.cn 
尿毒症尿液同样进行了凝胶色谱分离. 并将不同试样来源的 A 峰中分子物, 进行了离子交换 和反相高效液相色谱(HPLC)的多级分离. 运用基质辅助激光解吸电离-飞行时间质谱(MALDITOF MS)等分析手段, 证实了我们的方法对 A-3 亚组分内中分子物成功地进行了分离和纯化, 并确定了 A-3 亚组分内各中分子物的分子量.

\section{1 材料与方法}

\section{1 试样的来源}

12 例尿毒症患者血清及 12 例尿毒症患者尿样均由天津市第一中心医院提供, 患者均接受 血液透析治疗 0.5 2 a 不等, 其血液内肌酐含量均高于尿毒症临界值 707 ? $\mathrm{mol} / \mathrm{L}$, 患者日排尿 量在 1500 2000 mL之间, 接近正常人日排尿量. 4例正常人血样由天津市武警总队医院提供. 12 例正常人尿样由本所研究人员提供, 提供者在 1 个月内末服用任何药物.

\section{2 凝胶色谱及离子交换色谱分离}

尿毒症及正常人的血样经离心分离后, 取血清样在 PM10 膜上超滤, 以截留分子量大于 10,000 的大分子物质. 超滤血清分别在装有 Sephadex G15 的色谱柱上, 以 $0.01 \mathrm{~mol} / \mathrm{L} \mathrm{pH8.6}$ 的三乙胺-碳酸缓冲液作淋洗液进行凝胶色谱分离. 用分步收集器收集洗脱液, 收集的级分分 别在 $206 \mathrm{~nm}$ 处检测吸光值. 中分子组分经冷冻干燥后以备进一步分离. 正常人及尿毒症患者 的尿样在静置 $20 \mathrm{~min}$ 后, 于同样的条件下进行凝胶色谱分离和处理.

将来自正常人及尿毒症患者的尿液以及尿毒症血清的中分子组分干品, 用 $0.01 \mathrm{~mol} / \mathrm{L}$ pH8.6 的三乙胺-碳酸缓冲液溶解, 在 DEAE-Sephadex A25 的色谱柱上进一步分离, 以 0.01 $\mathrm{mol} / \mathrm{L} \mathrm{pH} 8.6$ 的三乙胺-碳酸缓冲液和 $0 \sim 0.2 \mathrm{~mol} / \mathrm{L} \mathrm{NaCl}$ 溶液作梯度洗脱. 用分步收集器收集洗 脱液, 收集的级分分别于 $230 \mathrm{~nm}$ 处检测吸光度, 被检测后的级分经冷冻干燥后留待后用.

凝胶色谱以及离子交换色谱的分离方法和条件详见我们以前的报道 ${ }^{[8]}$.

\section{$1.3 \mathrm{~A}-3$ 亚组分的脱盐及基质辅助激光解吸电离-飞行时间质谱(MALDI-TOF MS)分析}

将经凝胶色谱及离子交换色谱多步分离得到的不同试样来源的 A-3 亚组分, 分别在 0.4 $\mathrm{cm} \times 60 \mathrm{~cm}$ 装有 Sephadex G15 的色谱柱上, 以二次蒸馏水为淋洗液进行脱盐. 收集的级分于 $230 \mathrm{~nm}$ 处检测吸光度. 其操作方法和条件与前述的凝胶色谱分离相同.

将经上述分离和脱盐步骤得到的 A-3 亚组分, 在 BIFLE X III 型基质辅助激光解吸电离飞行时间质谱仪(Bruker Daltonics, 美国)上进行分析. 使用 $\mathrm{N}_{2}$ 激光器, 激光波长为 $337 \mathrm{~nm}$, 离 子检测电压为? $4.75 \mathrm{kV}$, 选用? -氧基-4-差基肉桂酸 $(\mathrm{CCA})$ 为基质, 以正离子方式记录谱图.

\section{4 反相 HPLC 法对 A-3 亚组分的分离}

经脱盐后的 A-3 亚组分的冷冻干燥品, 用二次蒸馏水将其溶解. 选择 $7.8 \mathrm{~mm} \times 300 \mathrm{~mm}$ Waters $\mathrm{G}_{8}$ 柱(? Bondapak)作为色谱柱,采用 Waters 600E 液相系统(美国)以及 Waters 996 紫外可见二极管阵列扫描检测系统(美国), 以 $0.2 \%$ 的三氟乙酸水溶液与乙腈的混合物作为流动相, $0.2 \%$ 的三氟乙酸水溶液与乙腈的比例为 $88: 12$. 流动相的流速为 $0.8 \mathrm{~mL} / \mathrm{min}$. 待色谱柱充分 平衡后, 每次进样 100 ? L, 以 245 或 $230 \mathrm{~nm}$ 处的紫外吸收为检测手段.

\section{2 结果与讨论}

\section{1 凝胶色谱分离}

在我们以前的文章中 ${ }^{[8]}$, 曾报道了凝胶色谱法对尿毒症血清、正常人血清及尿液的分离结 
果. 我们发现, 在洗脱体积为 $60 \sim 80 \mathrm{~mL}$ 区间内, 尿毒症血清及正常人尿液内均出现 2 个较大 的中分子峰. 按次序标记为中分子峰 $\mathrm{A}$ 和 $\mathrm{B}$. 而在正常人血清内仅在 $\mathrm{A}$ 峰位置有一个极小的 峰. 这就说明了 A，B 两峰, 是因肾衰而滞留在患者体内的具有异常高浓度的中分子物. 而正 常人可以通过尿液将其排于体外. 在以前的报道中还对不同试样来源的 B 峰中分子物进行了 离子交换色谱的分离和比较. 为了进一步考察患者通过尿液对中分子物的排除情况, 我们对 12 例尿毒症患者的尿液在相同条件下进行了凝胶色谱分离. 结果发现在洗脱体积为 $60 \sim 80 \mathrm{~mL}$ 区间内, 尿毒症患者的尿液也出现相似的 2 个中分子峰 $\mathrm{A}, \mathrm{B}$, 只是其浓度 (吸光值)要小于正常 人尿液. 因提供尿样的患者日排尿量接近于正常人, 故此患者尿液与正常人尿液内的中分子 含量具有一定的可比性. 这一结果说明了尿毒症患者亦能通过尿液部分地将中分子物排于体 外, 并非所有的中分子代谢废物都会因肾衰而滞留.

\section{$2.2 \mathrm{~A}$ 峰中分子物的离子交换色谱分离}

对凝胶色谱法得到的 $\mathrm{A}$ 峰中分子物, 我们进行了进一步的离子交换色谱分离. 图 1 是来自 尿毒症血清、正常人尿液以及患者尿液的 $\mathrm{A}$ 峰中分子物的典型分离结果. 比较图 1 中尿毒症 血清及正常人尿液的 $\mathrm{A}$ 峰中分子物的分离结果, 可以看出, 两种不同试样来源的 $\mathrm{A}$ 峰中分子 物均被分离成 9 个较突出的亚峰 A-1 A-9. 来自尿毒症血清 A 组分的各亚峰洗脱位置与来自 正常人尿液的各亚峰洗脱位置均能较好地重合. 这种相同的离子交换色谱行为显示出它们可 能具有相同的化学组成, 这进一步证实了我们以前根据两种来源的 A 峰中分子组分具有相似 的凝胶色谱、紫外和红外光谱行为, 而认为它们的组成相同或相似的判断 ${ }^{[8]}$.

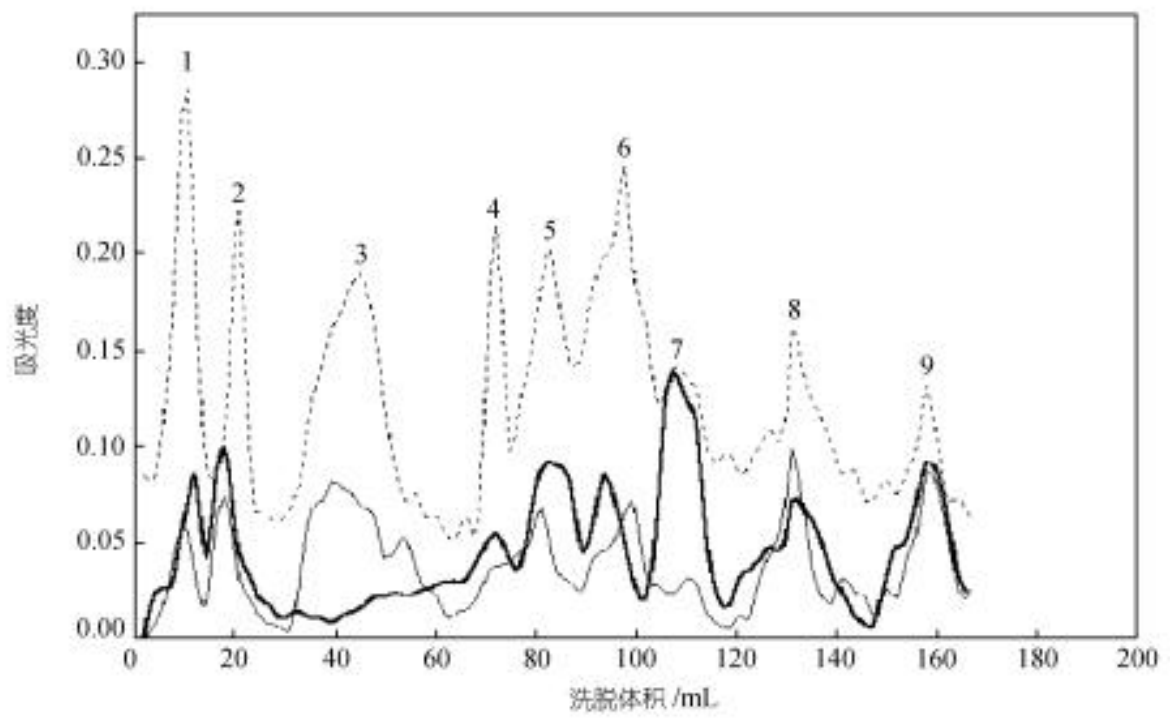

图 1 患者尿液(一一)、尿毒症血清(一一)以及正常人尿液(-----)的 $\mathrm{A}$ 峰中分子物的离子交换色谱

将图 1 中患者尿液的 $\mathrm{A}$ 峰中分子物的离子交换色谱与正常人尿液以及尿毒症血清的分离 结果进行比较, 可以看出, 患者尿液内缺少 A-3 亚峰, 而其他亚峰能同尿毒症血清和正常人尿 液相应的亚峰位置较好地重合. 这说明患者难以像正常人一样通过尿液将 A-3 亚峰中分子物 排于体外. 其他亚峰中分子物虽然也在患者体液内滞留, 却有一部分从尿液中排除. 这说明了 
A-3 亚峰中分子物是肾功能衰竭者不能通过尿液排除的中分子物, 因而这一组分的中分子物 在患者体内极易滞留和积累. 是否因此 A-3 组分就和肾功能衰竭的联系最为密切, 尚有待更进 一步的证实.

\subsection{A-3 亚峰中分子物的 MALDI-TOF MS 分析}

由于在离子交换色谱分离的步骤中, 淋洗液内加入了 $0 \sim 0.2 \mathrm{~mol} / \mathrm{L} \mathrm{NaCl}$ 梯度溶液, 使 A-3 亚组分内混有较多的 $\mathrm{NaCl}$ 盐分. 为了排除这些盐分对 A-3 亚组分的进一步分离和分析的影响, 将来自尿毒症血清及正常人尿液的 A-3 亚峰中分子物分别在 Sephadex G15 色谱柱上进行脱盐. 在脱盐过程中, 两种不同试样来源的 A-3 亚组分均被再一次分离成 2 个小组分, 按其洗脱的先 后次序, 标记为 A-3- I 和 A-3-II. 这表明脱盐步骤使 A-3 亚组分得到了进一步的分离和纯化. 必须指出的是, 这种凝胶色谱脱盐的方法除盐效率不够理想, 被分离出的 A-3- I 和 A-3- II 组 分内, 经检测仍含有一定量的 $\mathrm{NaCl}$, 但其含量尚不足以影响我们后续的分离和分析工作.

按照前面介绍的方法和条件, 我们对来自尿毒症血清及正常人尿液的 A-3-I 和 A-3-II 组 分都进行了 MALDI-TOF MS 分析, 结果见图 2 5.

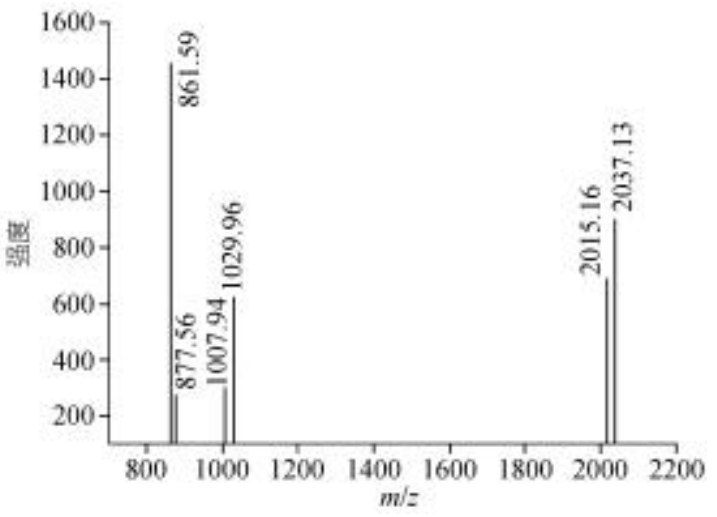

图 2 尿毒症血清 A-3- I 组分的 MALDI-TOF MS 分析结果

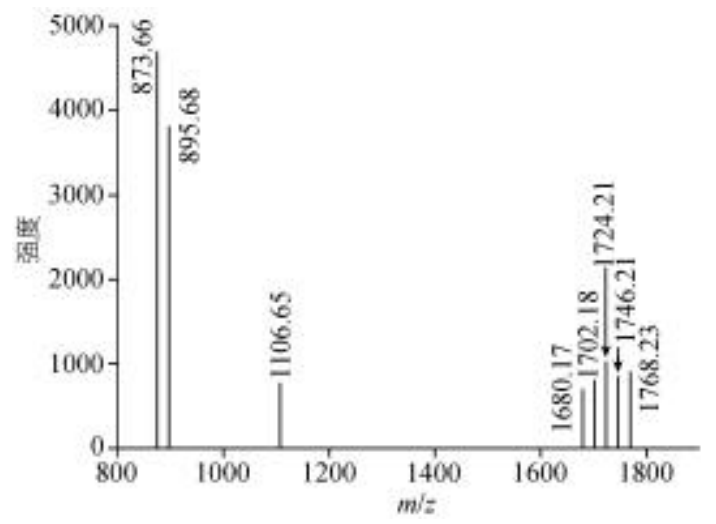

图 4 尿毒症血清 A-3- II 组分的 MALDI-TOF MS 分析结果

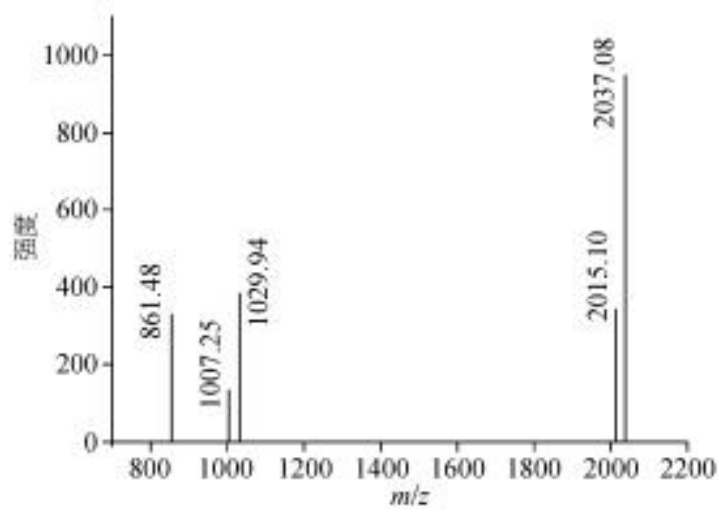

图 3 正常人尿液 A-3- I 组分的 MALDI-TOF MS 分析结果

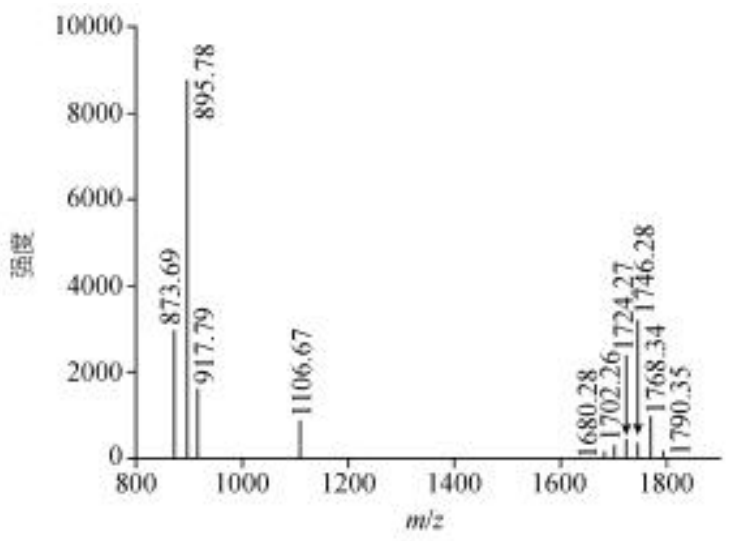

图 5 正常人尿液 A-3- II 组分的MALDI-TOF MS 分析结果 
图 2 和 3 分别是从尿毒症血清及正常人尿液中得到的 A-3- I 组分的 MALDI-TOF MS 分析 结果. 可以看出，来自尿毒症血清及正常人尿液的 A-3- I 组分的飞行时间质谱图上都出现了 3 组分子离子峰, 它们分别是: 第 1 组, 861.59, 877.56; 第 2 组, 1007.94, 1029.96; 第 3 组, 2015.16, 2037.13. 这 3 组峰表明在 A-3- I 组分内含有分子量为 $839\left(\mathrm{M}_{1}\right), 1007\left(\mathrm{M}_{2}\right)$ 及 $2015\left(\mathrm{M}_{3}\right)$ 的 3 种化 合物. $\mathrm{m} / \mathrm{z}$ 为 861.59 的分子离子峰, 是由化合物 $\mathrm{M}_{1}$ 的分子离子失去 1 个 $\mathrm{H}^{+}$同时结合 1 个 $\mathrm{Na}^{+}$ 形成的加合物 $\left[\mathrm{M}_{1} \mathrm{Na}\right]^{+}(839-1+23)$ 的假分子离子峰. 而 $\mathrm{m} / \mathrm{z}$ 为 877.56 的峰则是由 $\mathrm{M}_{1}$ 失去 1 个 $\mathrm{H}^{+}$后结合 1 个 $\mathrm{K}^{+}$形成的假分子离子 $\left[\mathrm{M}_{1} \mathrm{~K}\right]^{+}$峰. 第 2 组 $\mathrm{m} / \mathrm{z}$ 为 1007.94 和 1029.96 的 2 个峰则是 $\mathrm{M}_{2}{ }^{+}$和 $\left[\mathrm{M}_{2} \mathrm{Na}\right]^{+}$形成的 2 个峰, 证实了 $\mathrm{M}_{2}$ (1007)的存在. 同样, 第 3 组 $\mathrm{m} / \mathrm{z}$ 为 2015.16 及 2037.13 的峰是 $\mathrm{M}_{3}$ (2015) 失去 1 个电子后形成的 $\mathrm{M}_{3}{ }^{+}$以及与 $\mathrm{Na}^{+}$形成的加合物 $\left[\mathrm{M}_{3} \mathrm{Na}\right]^{+}$的分子离子峰. 表 明中分子化合物 $M_{3}$ (2015)的存在.

通过上述对图 2 和 3 的分析和比较, 可以看出, 来自尿毒症血清和来自正常人尿液的 A-3I 组分含有相同的化学物质, 即是 $M_{1}(M W, 839), M_{2}(M W, 1007)$ 和 $M_{3}(M W, 2015) 3$ 种中分子物. 另一方面, 从图 3 可以看出, 第 3 组峰最高, 第 2 组峰次之, 第 1 组峰最低, 这预示着正常人 尿液的 A-3- I 组分内, $M_{3}(\mathrm{MW}, 2015)$ 的含量最高, $\mathrm{M}_{2}(\mathrm{MW}, 1007)$ 的含量次之, 而 $\mathrm{M}_{1}(\mathrm{MW}, 839)$ 的含量最低. 同样, 根据图 2中的各组峰的相对高低, 可以看出在尿毒症血清的 A-3-I 组分内, $\mathrm{M}_{1}(\mathrm{MW}, 839)$ 的含量最高, $\mathrm{M}_{3}(\mathrm{MW}, 2015)$ 的含量次之, $\mathrm{M}_{2}(\mathrm{MW}, 1007)$ 的含量最低.

图 4 和 5 分别是来自尿毒症血清和来自正常人尿液的 A-3- II 组分的 MALDI-TOF MS 分析 结果. 图 4 和 5 也分别出现了相同的 3 组峰. 第 1 组, $\mathrm{m} / \mathrm{z}$ 为 $873.69,895.78,917.79$; 第 2 组, $\mathrm{m} / \mathrm{z}$ 为 1106.67 ; 第 3 组, $\mathrm{m} / \mathrm{z}$ 为 $1680.28,1702.26,1724.27,1746.28,1768.34,1790.35$. 第 $1 \sim 3$ 组峰分 别表明分子量为 873 的化合物 $\mathrm{M}_{4}$, 分子量为 1106 的化合物 $\mathrm{M}_{5}$ 和分子量为 1680 的化合物 $\mathrm{M}_{6}$ 的存在. 和前面对 A-3- I 组分的飞行时间质谱分析类似, 所有质谱峰, 只有 $\mathrm{m} / \mathrm{z}$ 为 873,1106 和 1680 三个峰是分别由化合物 $\mathrm{M}_{4}, \mathrm{M}_{5}$ 和 $\mathrm{M}_{6}$ 形成的真实分子离子峰, 其他的峰则是由 $\mathrm{M}_{4}$ 和 $\mathrm{M}_{6}$ 分别与不同数目的 $\mathrm{Na}^{+}$形成的加合物的假分子离子峰.

以上的结果表明, 来自尿毒症血清和来自正常人尿液的 A-3- II 组分都是由 $M_{4}(M W, 873)$ $\mathrm{M}_{5}(\mathrm{MW}, 1106)$ 和 $\mathrm{M}_{6}(\mathrm{MW}, 1680) 3$ 种化合物组成. 至此, 我们可以肯定, 来自尿毒症血清和正常 人尿液的 A-3 亚组分含有相同化学物质.

同样我们通过图 4 和 5 上各组峰的相对高低可以看出, 在正常人尿液和尿毒症血清的 A-3II 组分内, 化合物 $M_{4}, M_{5}$ 和 $M_{6}$ 的相对含量次序相同, 即 $M_{4}$ 含量最高, $M_{6}$ 含量次之, $M_{5}$ 含量 最低.

许多研究者在进行尿毒症中分子物分离时, 都借助已知分子量的化合物如 $\mathrm{V}_{\mathrm{B} 12}(\mathrm{MW}$, 1302)作为凝胶色谱分离时的分子量参照物, 以它们的洗脱时间或洗脱体积来标识凝胶色谱分 离的尿毒症中分子峰, 或确定尿毒症中分子物的分子量分布范围 ${ }^{[5,10]}$. 我们以前也曾采用这种 方法, 估计凝胶色谱分离得到的 A, B 两峰中分子物的分子量应在 700 1100 之间 ${ }^{[8]}$. 显然, 这 一估计与 A-3 亚组分的质谱分析结果相距甚远. 这是由于尿毒症中分子物的成分复杂多样, 要 找到化学组成和结构相似的分子量标准物是很困难的. 但我们也看到, 分子量在 839 2015 范 围内的 A-3 亚组分, 的确是出现在以分子量参照物标识的凝胶色谱中分子峰内. 这就说明 Fürst 等 ${ }^{[9]}$ 用以标识尿毒症中分子峰的方法是可行的. 


\section{$2.4 \mathrm{~A}-3$ 亚组分中分子物的反相 HPLC 分离}

来自正常人尿液的 A-3- I 和 A-3-II 组分反相高效液相色谱分离的结果分别见图 6 和 7. 从 图 6 和 7 可以看出, A-3-I 被又一次分离成 3 个相互独立的峰, A-3- II 组分也被分离成 3 个峰, 尽管在 A-3- II 组分的 HPLC 图谱上, 第 1 峰同第 2 峰稍有重叠, 但不影响我们从 HPLC 分离中 获取 A-3- II-1 和 A-3- II-2 两种组分. 根据前述 MALDI TOF MS 的分析结果, 我们已知 A-3I 组分内含有 $M_{1}(M W, 839), M_{2}(M W, 1007)$ 和 $M_{3}(M W, 2015) 3$ 种中分子化合物, A-3- II 组分内 含有 $M_{4}(M W, 873), M_{5}(M W, 1106)$ 和 $M_{6}(M W, 1680) 3$ 种化合物. 这就表明, A-3 组分内的 6 种中 分子物经反相 HPLC 的分离得到了彻底的分离和纯化, 在 HPLC 谱图上的每一个峰均代表一 种中分子化合物. 另一方面, 我们目前还不清楚这些中分子物的化学结构, 也未确定它们各自 的吸光系数, 故此难以通过比较 HPLC 谱图上的峰面积来确定各中分子物的含量或相对含量. 因此, 将 HPLC 分离的结果同 MALDI TOF MS 的分析结果比较, 还不足以确定 A-3- I-1, A-3| -2,A-3- | -3 同前面的 $M_{1}, M_{2}, M_{3}$ 之间, 以及 A-3- II-1, A -3- II -2, A - - II -3 同 $M_{4}, M_{5}, M_{6}$ 之间 的对应关系. 但是, MALDI-TOF MS 的分析结果与反相 HPLC 的分离结果都同样清楚地表明 A-3- I 含有 3 种中分子物, A-3- II 组分内亦含有 3 种不同的中分子物, 这就相互证明了我们的 分析和分离结果的可靠性. 进一步的结构测定将会明确 A-3- | -1, A - - - I-2, A-3- I-3 以及 A-3II -1,A-3- I| -2, A-3- II -3 与各分子量之间的对应关系.

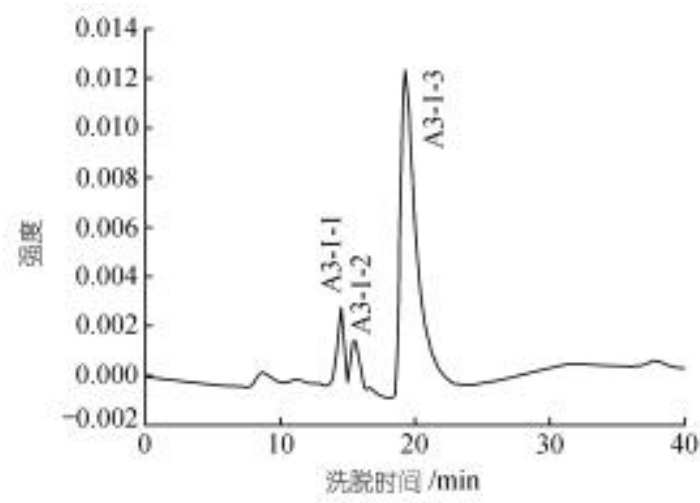

图 6 正常人尿液的 A-3- I 组分的反相高效液相 色谱

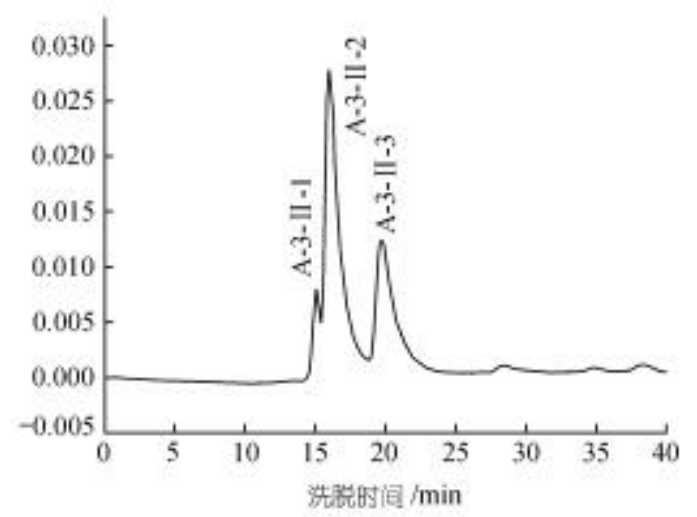

图 7 正常人尿液的 A-3- II 组分的反相高效液相 色谱

通过前述的多级分离, 至此, 未见前人报道的分子量为 $839,873,1007,1106,1680,2015$ 六种尿毒症中分子化合物已得到了分离和提纯, 这无疑为最终确定它们的化学结构以及病理 和生理作用机制铺平了道路.

\section{3 结论}

通过凝胶色谱、离子交换色谱和反相高效液相色谱, 对尿毒症患者及正常人的血清和尿液 的多级分离和比较. 从正常人尿液和尿毒症血清内, 得到了分子量为 $839,873,1007,1106$, 1680, 2015 六种未见前人报道的中分子化合物, 并对它们进行了彻底的分离和纯化. 我们的结 果证明了, 这 6 种中分子物是正常人可以通过尿液排于体外, 而肾衰患者却不能从尿液中将其 排除, 故而在患者血液内滞留和累积的重要成分. 虽然它们在患者体内的滞留究竟会导致哪 
些生理变化尚不清楚, 但这些“真正的”中等分子量代谢废物的分离和纯化, 为进一步鉴定它 们的化学结构，并最终揭示出它们的病理生理作用机制奠定了基础.

\section{参考文献}

1 Remond V, Reta D, Chen H, et al. Uremic toxicity: The middle molecule hypothesis resisted. Seminars in Nephrol, 1994, 14(3): 205 218

2 Remond V, Reta D, Psascale V, et al. Middle molecules: Toxicity and removal by hemodialysis and related strategies. Artif Organs, 1995, 19(11): 1120 1125

3 Gallice P. J Monti P, Braguer D L, et al. Identification of an ascorbic acid metabolite among " uremic middle molecules" . Clin Chem, 1990, 36(7): 1369 1372

4 Abiko $\mathrm{T}$, Onodera $\mathrm{T}$, Sekino $\mathrm{H}$ et al. A peptide isolation from the hemodialysate of a uremic patient with immunodeficiency inhibits lymphocyte stimulation. J Appl Biochem, 1981, 3: 562 569

5 吴兆龙，廖履坦，蔡菊辉，等. 尿毒症病人血清的中分子物质的生物活性. 中国病理生理学杂志, 1995, 11(1): 90 94

6 袁 直, 张 雯, 赵芬芝, 等. 尿毒症中分子的提取及初步分析. 高等学校化学学报, 1989, 10(4): 401 405

7 Chapman G V, Ward R A, Frrel P C, et al. Separation and quantification of “the middle molecules" in uremia. Kidney Int, 1980, 17: 82 88

8 储结根, 袁 直, 密怀风, 等. 尿毒症患者血清及正常人尿液内中分子物的分离分析. 中国科学, B 辑, 2000, 30(3): 250 255

9 Fü rst P, Zimmerman L, Bergstrom J. Determination of endogenous middle molecules in normal and uremic body fluids. Clin Nephrol, 1976, 5(4): 178 188

10 Gibson T P, Matusk E, Nelson L D, et al. Artifical kidneys and clearance calculations. Clin Pharmacol Ther, 1976, 20: 720 726 